\title{
Eine große Geschichte der direkten Demokratie in Deutschland
}

Wiegand, Hanns-Jürgen: Direktdemokratische Elemente in der deutschen Verfassungsgeschichte (Juristische Zeitgeschichte, Abt. I, Bd. 20), BWV Berliner Wissenschafts-Verlag, Berlin 2006, 631 Seiten, $€ 71$,-.

Hanns-Jürgen Wiegand baut seine verfassungsgeschichtliche Darstellung zunächst chronologisch auf: direktdemokratische Elemente in der Weimarer Republik, Volksabstimmungen im nationalsozialistischen Staat und direktdemokratische Elemente vor und bei der Entstehung des Grundgesetzes. Im sechsten Kapitel über direktdemokratische Elemente in der Verfassungsgeschichte der Bundesrepublik Deutschland, dem Herzstück der Arbeit (S. 237 bis 494, 721 Fußnoten), gliedert er auch systematisch: politische und rechtspolitische Auseinandersetzungen, die Grundzüge der wissenschaftlichen Diskussion, die Praxis in den Bundesländern - die Geschichte „der Normierung, Anwendung und Fortbildung direktdemokratischer Elemente“ auf Landesebene durchziehe die „Verzichts- und Vereitelungsgeschichte" auf der Bundesebene als besonderer, interdependenter Strang (vgl. S. 20, 238, 401) - und rechtliche Probleme der Volksgesetzgebung. Die letzten Kapitel über direktdemokratische Elemente in der Schweiz und in den USA sowie in der Europäischen Union und über „verfassungsrechtliche Erwägungen zu gegenwärtigen Gesetzentwürfen als Rahmen einer künftigen verfassungsgeschichtlichen Entwicklung auf der Ebene des Bundes“ runden den Band ab.

In seinem Vorwort hat der Verfasser ironisiert, Fachkollegen würden beim Aufspüren von Fehlern „vergnügliche Stunden verbringen“ (S. VI). Das ist nicht der Fall. Wiegand hat eine beeindruckende Fülle von Literatur durchgearbeitet, er hat einen gewaltigen Stoff gestaltet und daraus das große Bild eines dramatischen politischen und geistigen Ringens gezeichnet. Angesichts dieses Werkes zu beckmessern wäre töricht. Hier geht es auch nicht um Verästelungen; Wiegand legt ja nicht eine Zusammenfassung der beigezogenen Spezialliteratur vor, sondern sein Werk steht in der Tradition der „großen Erzählung“. Zu würdigen sind der weite Gedankenbogen, die umfassende Darstellung und die gelungenen Proportionen der Stoffbehandlung (zwei Aufgaben, an denen zum Beispiel unlängst Christopher Schwieger mit seiner Arbeit über „Volksgesetzgebung in Deutschland“ (1919 - 2002), die einfach die Landesebene ausklammerte, gescheitert ist ${ }^{3}$ ) sowie die klare und breite Perspektive.

Hervorzuheben ist die Sicherheit und Überzeugungskraft seines Urteils, gleichviel, ob der Verfasser historisch, politikwissenschaftlich oder staatsrechtlich argumentiert: Nach Wiegand „erwies sich die ,Mißerfolgsgeschichte' der Weimarer Volksgesetzgebung im Reich insbesondere als Erfolgsgeschichte der Vereitelungsmaßnahmen von Seiten ihrer Gegner" (S. 139). Dazu, wie etwa im Parlamentarischen Rat mit solchen historischen Tatsachen umgegangen wurde, bemerkt Wiegand gelassen: „Verfassunggebende Nationalversammlungen und Konvente sind keine wissenschaftlichen Kongresse. Politiker sind an wissenschaftliche Regeln und Methoden nicht gebunden.“ (S. 14) „Der Umgang mit Weimarer Verfassungstradition war letztlich politisch gewillkürt.“ (S. 233)

3 Allzu nachsichtig hierzu Julia Platter in ihrer Rezension in ZParl, 37. Jg. (2006), H. 3, S. 654 f. (S. 655, Fn. 1). Wiegand hat seinerseits den kommunalen Bereich (Bürgerbegehren und Bürgerentscheid) nur gelegentlich herangezogen (vgl. S. 21), aber dies erscheint mit Rücksicht auf den ohnehin erreichten Umfang des Werks verzeihlich. 
Im Abschnitt „Grundzüge der wissenschaftlichen Diskussion“ liefert Wiegand eine „Literatur-“, „Meinungs-“ und „Dogmengeschichte“ (S. 318) der Staatsrechtslehre über fast sechs Jahrzehnte und das gleiche noch einmal für die Politikwissenschaft, strukturiert nach Dekaden, wissenschaftlichen Generationen (mit oder ohne persönliche Weimar-Erfahrung), akademischen Lehrer-Schüler-Verhältnissen, fachlichen Ansätzen und Argumentationstypen. Bei dem komplexen Stoff zweier Fachgeschichten zu diesem Spezialthema hilft das Personenregister des Anhangs. Wiegand zeichnet den „Perspektivenwechsel“ in den achtziger Jahren nach (S. 345-353) und konstatiert für die letzten Jahre, von einer argumentativen PattSituation könne „nicht mehr gesprochen werden. Die rechtspolitische und die verfassungsrechtliche Argumentationslast hat sich eindeutig zugunsten der Befürworter verschoben“ (S. 400). So habe zum Beispiel die Praxis in den Ländern und Kommunen „bisher die ,Destabilisierungs-' und die ,Fragmentierungstheorie' nicht bestätigt“" (S. 439).

Die ,rechtlichen Probleme der Volksgesetzgebung" analysiert Wiegand vor allem anhand acht großer Entscheidungen der Landesverfassungsgerichte zu Verfahrensreformen aus den Jahren 1997 bis 2002 und wird dabei, wo es ihm geboten erscheint, so scharf wie präzise: Nachdem er beispielsweise beim Thüringer Verfassungsgerichtshof (Urteil vom 19. September 2001) dessen „- wenig seriösen - Umgang mit Erfahrungen der deutschen Verfassungsund Zeitgeschichte" (S. 487) ebenso angeprangert hat wie seine "- methodisch und inhaltlich prekäre(n) - Vermischung verfassungsdogmatischer und verfassungspolitischer Gesichtspunkte“, fasst er zusammen: „Das erkenntnisleitende Interesse der Verfassungsrichter bestand in der Stärkung und Absicherung der Position des Landtages und der Landesregierung und ihrer Funktionsträger, d. h. der Mitglieder der Führungsgruppen der gewählten politischen Parteien." (S. 487)

Besonders fruchtbar erscheint Wiegand der komparatistische Ansatz. Es gehöre zu den Versäumnissen der deutschen Verfassungsgeschichtsschreibung, „die neuere schweizerische Verfassungsgeschichte kaum berücksichtigt und vergleichend herangezogen zu haben. ... Gerade aus schweizerischer Sicht wird der schwere Stand deutlich, den die deutschen Befürworter nicht nur zur Zeit des monarchischen Konstitutionalismus, sondern auch in den nachfolgenden Epochen des republikanischen demokratischen Repräsentativsystems hatten. Obrigkeitliche Staatsauffassung und Verfassungstradition wirkten noch lange nach und verbanden sich mit dem Interesse an einer stabilen Parteienherrschaft." (S. 503 f.) Die komparatistischen Erkenntnisse aus der Schweiz und den US-Bundesstaaten hätten die Gegner „in Argumentations- und Beweisnot gebracht und ihre negativen Positionen erschüttert" (S. 508).

Dass dieses Werk meisterlich erscheint, ist kein Zufall, sondern hängt mit der Person des Autors zusammen. Dies ist nicht die Erstlingsschrift eines begabten jungen Menschen, sondern Wiegand, Jurist und promovierter Theologe (F.-J.-Stahl-Experte), hat am Ende seines siebten Lebensjahrzehnts sein Magnum opus vorgelegt (und damit seinen zweiten - gesellschaftswissenschaftlichen - Doktorhut geholt). Daher rührt die Souveränität der Problembehandlung, die methodische Versiertheit und - man kann es nicht anders nennen - die Schönheit der Darstellung. Bemerkenswert erscheint auch, dass Wiegand kein „Kombattant" ist; er hat in den einschlägigen wissenschaftlichen Kontroversen der letzten 20 Jahre gerade nicht mitgestritten. Gewiss zeigt er sich für seinen Gegenstand aufgeschlossen - er spricht am Ende von „Verständnis“ und „Empathie“ (S. 558) -, aber sein Ziel ist doch, „traditionelle, historisch begründete Meinungen von verfassungsinterpretatorischer und verfassungspolitischer Relevanz kritisch zu überprüfen" (S. 19). Daher verleiht ihm der 
Umstand, dass er nicht in die früheren Auseinandersetzungen involviert war, auch eine gewisse Unbefangenheit, was seine in diesem Werk erarbeiteten Urteile umso wertvoller macht.

Wiegand hat eine große Geschichte der direkten Demokratie in Deutschland geschrieben, ein grundlegendes Werk für jede weitere Beschäftigung mit der Materie. Die Spezialliteratur hat daneben immer noch ihr Recht.

Otmar Jung

\section{Direkte Demokratie in Schweizer Städten: empirische Bestandsaufnahme}

Bützer, Michael: Direkte Demokratie in Schweizer Städten. Ursprung, Ausgestaltung und Gebrauch im Vergleich (Reihe „Studien zur Sachunmittelbaren Demokratie“, Band 4), Nomos Verlagsgesellschaft, Baden-Baden 2007, 308 Seiten, $€ 49,-$.

Dass Recht und Praxis unmittelbarer Demokratie gemeinhin vor allem für die übergemeindlichen Ebenen behandelt werden, ist kein Alleinstellungsmerkmal der Schweiz. Allerdings kommt dort hinzu, dass sich die einschlägigen Rechtsgrundlagen nicht nur von Kanton zu Kanton, sondern auch unter den jeweiligen (Einwohner-)Gemeinden maßgeblich unterscheiden. Vor diesem Hintergrund gibt die Arbeit von Michael Bützer, die 2006 von der Philosophischen Fakultät der Universität Zürich als Dissertation angenommen wurde, hinsichtlich der 118 (geographischen) Städte mit mehr als 10.000 Einwohnern umfassend Auskunft. Weitgehend ausgeklammert bleiben Formen der Versammlungsdemokratie, wobei aber aufgezeigt wird, dass die durchschnittliche Teilnahme an Bürgerentscheiden deutlich höher liegt als diejenige an Versammlungen. Der Autor bilanziert deshalb, Volksabstimmungen stellten „ganz eindeutig geeignetere Instrumente als Bürgerversammlungen dar, um die demokratische Qualität von kommunalpolitischen Entscheiden zu gewährleisten" (S. 71).

Bützers Untersuchung gliedert sich in zwei Teile: Zunächst werden die politischen Institutionen, vor allem die bestehenden Einrichtungen direkter Demokratie, und anschließend der jeweilige Abstimmungsgebrauch analysiert. Dabei bezieht sich die Arbeit in beiden Teilen auf die Jahre 1990 bis 2000.

Im ersten Teil folgen auf einen Überblick zu den kommunalverfassungsrechtlichen Rahmenbedingungen Angaben zu Verbreitung und Ausgestaltungen von obligatorischen und fakultativen Referenden, Initiativen und Gegenvorschlägen in den einzelnen Städten. Diese werden durch Kurzbeschreibungen zu den Rechtslagen in den Kantonen ergänzt, die im Anhang abgedruckt sind. Dabei stellt der Autor die Materie umfassend dar und analysiert Entwicklungstendenzen während des Untersuchungszeitraums. Er fragt auch nach dem direktdemokratischen Öffnungsgrad der politischen Systeme. Diesen macht er zum einen an der materiellen Reichweite der Volksrechte, zum anderen an ihren formellen Erfordernissen fest. Hierbei mahnt er wegen der vereinfachten Zusammenhänge zwar zur Vorsicht, doch kann immerhin ein recht deutlicher Zusammenhang zwischen Öffnungsgraden und Rechtstraditionen aufgezeigt werden. Bützer unterscheidet hier zwischen deutschschweizer Gemeinden mit Versammlungssystem (Landgemeinden) beziehungsweise Stadtgemeinden 